\title{
Chern-Simons action for zero-mode supporting gauge fields in three dimensions
}

\author{
C. Adam* \\ Wolfgang-Pauli Institute clo Institute of Mathematics, Vienna University, 1090 Vienna, Austria \\ B. Muratori ${ }^{\dagger}$ \\ CERN, SL-AP Division, Geneva 32, CH-1211, Switzerland \\ C. Nash \\ Department of Mathematical Physics, National University of Ireland, Maynooth, Ireland
}

(Received 29 November 2002; published 28 April 2003)

\begin{abstract}
Recent results on zero modes of the Abelian Dirac operator in three dimensions support to some degree the conjecture that the Chern-Simons action admits only certain quantized values for gauge fields that lead to zero modes of the corresponding Dirac operator. Here we show that this conjecture is wrong by constructing an explicit counterexample.
\end{abstract}

DOI: 10.1103/PhysRevD.67.087703

PACS number(s): 11.15.Tk

In the last few years a considerable amount of interest has been devoted to the study of zero modes of the Abelian Dirac operator in three-dimensional Euclidean space, that is, to square-integrable solutions of the Dirac equation

$$
\not D \Psi \equiv \vec{\sigma}[i \vec{\partial}+\vec{A}(\vec{x})] \Psi(\vec{x})=0
$$

where $\vec{x}=\left(x_{1}, x_{2}, x_{3}\right), \vec{\sigma}$ are the Pauli matrices, and $\Psi$ is a two-component spinor. In addition, the gauge field $\vec{A}$ is assumed to obey certain integrability conditions (e.g., square integrability of the related magnetic field $\vec{B}=\vec{\partial} \times \vec{A}$ ).

On the one hand, such solutions are relevant for the quantum mechanical behavior of nonrelativistic electrons (see e.g., Ref. [1]), because solutions to the above equation are, at the same time, solutions to the Pauli equation (the Pauli equation is obtained by just squaring the Dirac operator in the above equation, i.e., $\mathbb{D}^{2} \Psi=0$ ). On the other hand, solutions to the Dirac equation are also relevant for (Euclidean) quantum electrodynamics, as was discussed, e.g., in Refs. $[2,3]$.

Some first examples of zero modes were constructed in Ref. [4]. In Ref. [5] a class of Dirac operators and their zero modes was constructed which depend on a function that is arbitrary up to certain boundary conditions, thereby relating the existence of these zero modes to some topological condition. Some further examples of zero modes were given in Ref. [6] and in Ref. [7]. In Refs. [8,9] the first examples of Dirac operators with multiple zero modes were given, thereby demonstrating the existence of the phenomenon of zero mode degeneracy. Further, a relation between the number of zero modes and a certain topological linking number (the Hopf index) of the corresponding gauge field was established in Ref. [9]. A very detailed and more geometrical dis-

\footnotetext{
*Email address: adam@mat.univie.ac.at

†Email address: Bruno.Muratori@cern.ch

‡Email address: cnash@stokes2.thphys.may.ie
}

cussion of these Dirac operators with multiple zero modes, based on the concept of Riemannian submersions, was given in Ref. [10].

In Ref. [11] the following two results were proved: (i) For the one-parameter family of gauge potentials $t \vec{A}$ zero modes may exist for at most a finite set of values $t_{i}$ for any $t$ $\in\left(t_{0}, t_{1}\right)$, and (ii) The set of gauge potentials with no zero modes is a dense subset of the set of all gauge potentials (with certain decay properties). Recently, some results on the dimensionality of the space of gauge potential with zero modes were obtained in Ref. [12]. There it was proven that locally the space of gauge potentials with (at least) one zero mode is of co-dimension one within the space of all gauge potentials (with certain decay properties). In addition, some results on the dimensionalities of spaces of gauge potentials with multiple zero modes were proven.

The above-described results would suit well with the assumption that there exists a certain functional of the gauge potential which may admit only fixed or quantized values for gauge potentials that support zero modes. The simplest functional one can imagine is the Chern-Simons action, which has the additional attractive feature of being a topological invariant (i.e., independent of the metric). Therefore, if the existence and degeneracy of zero modes is related to some topological features, as was speculated, e.g., in Ref. [3], the Chern-Simons action would be an obvious candidate.

In addition, the assumption of quantized Chern-Simons action for gauge potentials with zero modes is further supported by the results of Ref. [9], where a whole class of gauge potentials with an arbitrary number of zero modes was constructed. For all these gauge potentials, which are characterized by an arbitrary function and an integer $l$ (the number of zero modes for a given gauge potential), the ChernSimons action indeed admits only the quantized values

$$
\frac{1}{16 \pi^{2}} \int d^{3} x \vec{A} \cdot \vec{B}=\frac{1}{4}\left(l+\frac{1}{2}\right)^{2}
$$

where $l$ is the number of zero modes for the given gauge 
potential (the Chern-Simons action for all the gauge potentials of Ref. [9] was calculated explicitly in Ref. [13]).

(Remark: In Ref. [9] a fixed universal background gauge potential was added to all the gauge potentials, $\vec{A} \rightarrow \overrightarrow{\widetilde{A}}=\vec{A}$ $+\vec{A}^{\text {b }}$, in order to relate the resulting gauge potentials $\overrightarrow{\widetilde{A}}$ to Hopf maps. For these resulting gauge potentials the ChernSimons action is automatically quantized, $\left(1 / 16 \pi^{2}\right) \int d^{3} x \overrightarrow{\widetilde{A}}$ $\cdot \overrightarrow{\widetilde{B}}=(1 / 4)(l+1)^{2}$, because the integer Hopf index is given by the Chern-Simons action. However, even the original zero-mode supporting gauge potentials, without the background field, lead to the quantized Chern-Simons action (2), although they cannot be directly related to Hopf maps.)

Another, more general argument in favor of quantized Chern-Simons action for zero-mode supporting gauge fields is related to the anomaly equation in Minkowski space,

$$
\partial^{\mu} J_{\mu}=\frac{1}{16 \pi^{2}} \epsilon^{\mu \nu \alpha \beta} F_{\mu \nu} F_{\alpha \beta} .
$$

Here, $J_{\mu}$ is the current density of a chiral (left-handed) Weyl Fermion which couples to the Abelian gauge field $A_{\mu}$ with field strength $F_{\mu \nu}$. Choosing the gauge $A_{0}=0$ and integrating the above equation over all space and over the finite time interval $\left[t_{i}, t_{f}\right]$ results in the equation

$$
\begin{gathered}
Q\left(t_{f}\right)-Q\left(t_{i}\right)=-\frac{1}{4 \pi}\left[I_{\mathrm{CS}}\left(t_{f}\right)-I_{\mathrm{CS}}\left(t_{i}\right)\right] \\
Q(t) \equiv \int d^{3} x J_{0}(t, \vec{x}), \quad I_{\mathrm{CS}}(t) \equiv \int d^{3} x(\vec{A} \cdot \vec{B})(t, \vec{x})
\end{gathered}
$$

where the left-hand side of Eq. (4) is the change in particle number between $t_{\mathrm{i}}$ and $t_{\mathrm{f}}$ [13]. If we further assume that the change in particle number, $Q\left(t_{f}\right)-Q\left(t_{i}\right)$, is two times the number of levels (zero modes) $L$ crossed in the (adiabatic) change from $\vec{A}\left(t_{i}, \vec{x}\right)$ to $\vec{A}\left(t_{f}, \vec{x}\right)$ - as is usually assumedthen the equation

$$
2 L=-\frac{1}{4 \pi}\left[I_{\mathrm{CS}}\left(t_{f}\right)-I_{\mathrm{CS}}\left(t_{i}\right)\right]
$$

results. Consequently, $L$ must be independent of the path $\vec{A}(t, \vec{x})$ which connects $\vec{A}\left(t_{i}, \vec{x}\right)$ and $\vec{A}\left(t_{f}, \vec{x}\right)$ (see Ref. [13] and the literature cited there for details). Obviously, Eq. (6) is automatically satisfied when level crossing may occur only for fixed, quantized values of the Chern-Simons action $I_{\mathrm{CS}}$. Therefore, Eq. (6) is compatible with the assumption that zero modes only exist for certain quantized values of the Chern-Simons action. The correctness of this assumption would, in fact, be the simplest way to realize Eq. (6).

All in all, the existing results on zero modes provide some evidence for the assumption that the Chern-Simons action is quantized for gauge potentials with zero modes. Therefore, a further investigation of this question is of some interest. Topologically the Chern-Simons action corresponds to a cohomology class in $H^{3}\left(S^{3} ; \mathbf{R} / \mathbf{Z}\right)$ and this is not constrained to be discrete. In the remainder of the paper we want to demon- strate that the above assumption is incorrect in the general case by constructing a simple counterexample.

For this purpose, let us briefly review some results from Ref. [5]. There it was shown that the ansatz $(r \equiv|\vec{x}|)$

$$
\Psi=g(r) \exp \left(i f(r) \frac{\vec{x}}{r} \vec{\sigma}\right)\left(\begin{array}{l}
1 \\
0
\end{array}\right)
$$

for the spinor leads to a zero mode for the gauge field

$$
A_{i}=h(r) \frac{\Psi^{\dagger} \sigma_{i} \Psi}{\Psi^{\dagger} \Psi}
$$

provided that $g(r)$ and $h(r)$ are given in terms of the independent function $f(r)$ as $\left({ }^{\prime} \equiv d / d r\right)$

$$
\begin{gathered}
g^{\prime}=-\frac{2}{r} \frac{t^{2}}{1+t^{2}} g . \\
h=\left(1+t^{2}\right)^{-1}\left(t^{\prime}+\frac{2}{r} t\right)
\end{gathered}
$$

where

$$
t(r):=\tan f(r) .
$$

Here, a sufficient condition on $t(r)$ leading to smooth, nonsingular and $L^{2}$ spinors and smooth, nonsingular gauge potentials with finite energy $\left[\int(\vec{B})^{2}\right]$ and finite Chern-Simons action $\left(\int \vec{A} \vec{B}\right)$ is

$$
\begin{gathered}
t(0)=0, \quad t(r) \sim c_{1} r+O\left(r^{2}\right) \quad \text { for } r \rightarrow 0, \\
t(\infty)=\infty .
\end{gathered}
$$

Further, the Chern-Simons action for this class of gauge fields may be expressed like

$$
\begin{aligned}
\int d^{3} x \vec{A} \cdot \vec{B}= & 4 \pi(2 \pi-4) \int_{0}^{\infty} d r \frac{r t h^{2}}{1+t^{2}} \\
& +4 \pi(-2 \pi+8) \int_{0}^{\infty} d r \frac{r^{2} t^{\prime} h^{2}}{1+t^{2}} \\
= & 4 \pi \int_{0}^{\infty} \frac{d r}{r}\left(r f^{\prime}+\sin 2 f\right)^{2}\left[(-2 \pi+8) r f^{\prime}\right. \\
& +(\pi-2) \sin 2 f]
\end{aligned}
$$

where spherical coordinates $(r, \theta, \varphi)$ were introduced and the angular integrations have already been performed.

Next, let us introduce the one-parameter family of functions

$$
t_{a}(r)=r\left(a+r^{2}\right)
$$

where $a$ is an arbitrary real number. $t_{a}$ obeys the integrability conditions (12) and (13) for all values of $a$, therefore $t_{a}$ 
defines a zero-mode supporting gauge potential and a wellbehaving zero mode for all values of $a$. We easily compute

$$
t_{a}^{\prime}=a+3 r^{2}, \quad h_{a}=\frac{3 a+5 r^{2}}{1+r^{2}\left(a+r^{2}\right)^{2}}
$$

and

$$
\begin{aligned}
\int d^{3} x \vec{A} \cdot \vec{B}= & 16 \pi \int_{0}^{\infty} d r r^{2}\left[a+(5-\pi) r^{2}\right] \\
& \times \frac{\left(3 a+5 r^{2}\right)^{2}}{\left[1+r^{2}\left(a+r^{2}\right)^{2}\right]^{3}} .
\end{aligned}
$$

This last integral may be easily evaluated numerically with the help of Mathematica. Before presenting the result of the numerical integration, we want to remark that, in the limit $a \rightarrow+\infty, t_{a}$ is equal to the simplest case $t(r)=r$ up to an (infinite) rescaling, which does not change the value of the Chern-Simons action. Further, the simplest gauge potential with $t=r$ is, at the same time, the simplest gauge potential of the class of gauge potentials constructed in Ref. [9], with one zero mode (i.e., $l=1$ ). The resulting Chern-Simons action may be evaluated with the help of Eq. (2), and leads to

$$
\int d^{3} x \vec{A} \cdot \vec{B}=16 \pi^{2} \frac{1}{4}\left(\frac{3}{2}\right)^{2}=9 \pi^{2} .
$$

Therefore, the Chern-Simons action (16) normalized according to $\left(9 \pi^{2}\right)^{-1} \int d^{3} x \vec{A} \cdot \vec{B}$ should approach the value one in the limit of large positive $a$.

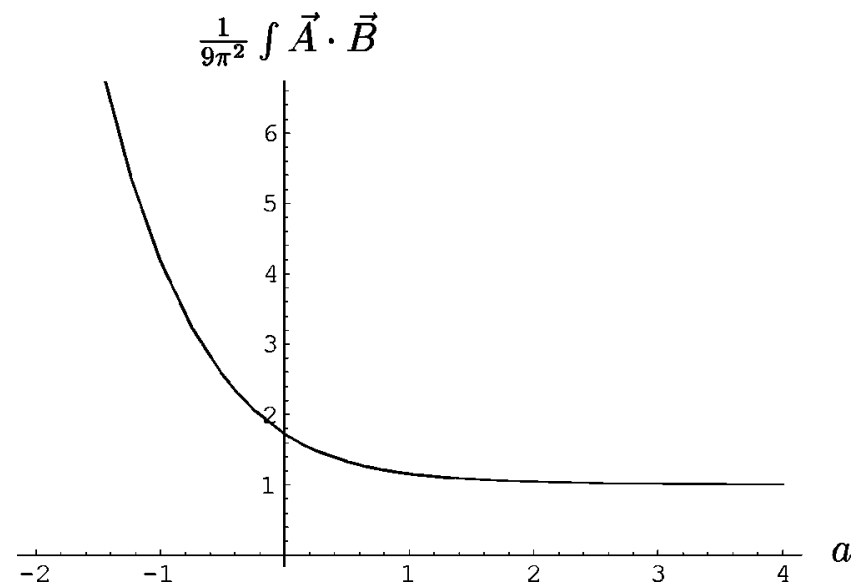

FIG. 1. The appropriately normalized Chern-Simons action $\left(1 / 9 \pi^{2}\right) \int \vec{A} \cdot \vec{B}$ as a function of the parameter $a$.

In Fig. 1 we plot the Chern-Simons action (16) multiplied by $\left(9 \pi^{2}\right)^{-1}$, as a function of $a$. For $a \rightarrow+\infty$ it indeed approaches the value 1 . Further, it increases for decreasing values of $a$ and reaches arbitrarily large values for sufficiently negative values of $a$. Obviously, the Chern-Simons action in Fig. 1 may admit all values greater than 1 . This result demonstrates that the Chern-Simons action for gauge potentials with zero modes is, in general, not quantized, which was the purpose of this brief report.

The authors thank R. Jackiw for helpful comments. Further, C.A. acknowledges support from the Austrian START award project FWF-Y-137-TEC of N.J. Mauser.
[1] J. Fröhlich, E. Lieb, and M. Loss, Commun. Math. Phys. 104, 251 (1986).

[2] M.P. Fry, Phys. Rev. D 54, 6444 (1996).

[3] M.P. Fry, Phys. Rev. D 55, 968 (1997); 56, 6714(E) (1997).

[4] M. Loss and H.-T. Yau, Commun. Math. Phys. 104, 283 (1986)

[5] C. Adam, B. Muratori, and C. Nash, Phys. Rev. D 60, 125001 (1999).

[6] D.M. Elton, J. Phys. A 33, 7297 (2000).

[7] C. Adam, B. Muratori, and C. Nash, Mod. Phys. Lett. A 15, 1577 (2000).
[8] C. Adam, B. Muratori, and C. Nash, Phys. Lett. B 485, 314 (2000).

[9] C. Adam, B. Muratori, and C. Nash, Phys. Rev. D 62, 085026 (2000).

[10] L. Erdos and J.P. Solovej, Rev. Math. Phys. 13, 1247 (2001).

[11] A.A. Balinsky and W.D. Evans, J. Funct. Anal. 179, 120 (2001).

[12] D.M. Elton, Commun. Math. Phys. 229, 121 (2002).

[13] C. Adam, B. Muratori, and C. Nash, Phys. Rev. D 62, 105027 (2000). 